\title{
The Prevalence and Severity of Depression, Anxiety and Stress as Well as Coping Strategies Used by Medical Undergraduate Students Enrolled in Arsi University: A Cross-Sectional Study
}

Leta Melaku ( $\nabla$ letamelaku@gmail.com ) Arsi University College of Health Sciences

Guta Bulcha

Arsi University College of Health Sciences

Deressa Worku

Arsi University College of Health Sciences

Research

Keywords: Depression, Anxiety, Stress, Coping Strategies, Medical students, Arsi University

Posted Date: January 27th, 2021

DOl: https://doi.org/10.21203/rs.3.rs-152511/v1

License: (c) (1) This work is licensed under a Creative Commons Attribution 4.0 International License.

Read Full License 


\section{Abstract}

\section{Background}

Every individual has a certain number of coping resources, and once these coping resources are challenged, mental illness usually results.

\section{Objective}

We aimed to determine the prevalence and severity of depression, anxiety and stress as well as coping strategies used by medical undergraduate students enrolled in Arsi University.

Methods

Institutional based cross-sectional design was conducted on 265 sampled medical students. Participants were selected by systematic random sampling technique. Data were collected by pre-tested, structured and self-administrated questionnaire. Afterwards, logistic regression analysis was employed and statistical significance was accepted at $p<0.05$.

Result

In the present study 5 questionnaires were rejected for incompleteness, resulting in a response rate of $98.1 \%$. The current prevalence rate of depression, anxiety and stress were $52.3 \%, 60.8 \%$ and $40.4 \%$ respectively. The proportion of respondents who had extremely severe symptoms of depression, anxiety and stress were $6.2 \%, 16.2 \%$ and $2.3 \%$ respectively. Depression was specifically associated with a monthly income and residency. Anxiety was significantly associated with residency and educational level. Stress was associated with a monthly income, educational level and residency. The main coping strategy adopted by respondents was religion. In particular, the coping mechanisms of denial, substance use, behavioral disengagement, venting, self-blame were significantly associated to DAS.

\section{Conclusion}

Overall prevalence rate of depression, anxiety and stress is alarmingly high. Therefore, implementing stress reduction interventions and establishing student counseling center is highly recommended.

\section{Introduction}

Stress is a biopsychosocial model that refers to the consequence of failure of an organism to respond adequately to mental, emotional or physical demands ${ }^{1}$. Clinically, anxiety is characterized by intense feeling of dread, accompanied by somatic symptoms that indicate a hyperactive autonomic nervous system $^{2}$. Whereas, depression manifests as loss of interest or pleasure, sadness, feelings of guilt or low self-worth, disturbed sleep or appetite, extreme tiredness, and poor concentration ${ }^{3}$. Studies suggest that mental health worsens after students begin medical school and remains poor throughout training. 
Consequently, it is commonly observed that medical students and resident doctors experience high rates of psychological morbidity when compared with students in other disciplines ${ }^{4}$. Previous studies have shown that stress is adversely affecting medical student physical and cognitive capacities. When associated with anxiety and depression, occupational stress, as experienced by the student, can influence his or her quality of life and decrease his or her academic performance due to anxiety-induced difficult cognitive functioning, such as memory disorders, blockage, incapacity to make decisions, and increased sensitivity to appraisals of others ${ }^{5}$. Similarly, high rates of depression, anxiety, and stress can result in poor quality of life, drug abuse and suicide ${ }^{6}$. Globally, the incidence of depression, anxiety and stress among medical students is increasingly reported. For instance, the prevalence of stress within undergraduate medical students of United States $(26 \%)^{7}$, Britain $(31.2 \%)^{8}$, Malaysia $(41.9 \%)^{9}$, Nigeria $(94.2 \%)^{10}$ and Ethiopia $(52.4 \%)^{11}$. Sources of increased stress levels experienced by students include overcrowded lecture halls, semester grading system, inadequate resources and facilities, adaptation to the new environment, being away from home for the first time, and changes in living arrangements, vastness of syllabus, long hours and expectations of rote learning ${ }^{12-14}$.

Coping strategies are specific efforts that individuals employ to manage stress, both behavioral and psychological, so they can tolerate, reduce, or minimize stressful events. Although people differ in the way they think about and react to the stressful situations, coping plays a central role in adaptation to stressful life events ${ }^{15}$. Every individual has a certain number of coping resources, and once these coping resources are challenged or exceeded, stress usually results ${ }^{10}$. The equipping of undergraduates with the skills necessary to recognize personal distress and to develop strategies to promote their own well-being is fundamental to promoting professionalism ${ }^{16}$. Conversely, failure in coping stress may lead students to stop or discontinue further education, suicidal intentions or make students participating in activities like smoking, drinking, aggressive behavior with others, damaging institution's property or involving in violent activities by disrespecting laws and right of others ${ }^{2,17}$. Therefore, the main aim of this study was to determine the prevalence and severity of depression, anxiety and stress as well as coping strategies used by medical undergraduate students enrolled in Arsi University.

\section{Methods}

Institutional based cross-sectional design was conducted in Arsi University from January 03-31, 2019 among 265 sampled undergraduate medical students. The sample size was first estimated by single population proportion, thereafter, through a correction formula since overall numbers of medical students in study area were $<10,000$. After proportional allocation of the calculated sample size to each academic year level, stratified random sampling was employed. The required respondents were chosen from each stratum by randomly selecting the first respondent based on their id number, and then every 4 intervals through systematic random sampling. All medical students in Arsi University were eligible to participate with exception to severely ill students and that were out of town during the time of data collection. Data were collected by self-administered a questionnaire that comprises the following parts. 
1. Sociodemographic profile: includes age, gender, enrollment year, marital status, residence, income, religion and ethnicity.

2. Depression, Anxiety, Stress Scale - 21 (DASS-21): is a self-report tool designed to measure the emotional states of depression, anxiety and stress over the last week ${ }^{18}$. Each of the three DASS-21 scales contains 7 items. The responses are given on a 4-point Likert scale, ranging from zero if "I strongly disagree" to 3 if "I totally agree". According to DASS-21 scoring instructions, the obtained DASS-21 scores need to be multiplied by 2 to have the final score and the results correspond to levels of symptoms, ranging from "normal" to "extremely serious" (Table 1). However, to determine the prevalence of DAS, DASS-21 scores with normal levels of depression, anxiety, and stress were coded as " 0 " whereas those with mild, moderate, severe, or extremely severe levels were coded as " 1. " Various studies have shown the strong internal consistency of the DASS-21 19,20 .

3. Brief COPE scale: is designed to assess a number of different coping behaviors and thoughts after a person's response to a specific situation among adults with or without clinical conditions ${ }^{21}$. It consists of 28 items and each item is rated on a 4-point Likert scale ranging from "I have not been doing this at all (score 1)" to "I have been doing this a lot (score 4)". The items were scored to produce 14 dimensions (minimum mean score was 2 and maximum score was 8 ) and each dimension reflects the use of a coping strategy such as active coping, planning, acceptance, denial, self-distraction, use of substance, use of emotional support, use of instrumental support, behavioural disengagement, venting, positive reframing, humour, religion, and self-blame ${ }^{21}$. Mean score interpretations were as follows: $2.00=$ haven't been doing this at all, 2.01 to $4.00=$ have been doing this a little bit, 4.01 to $6.00=$ have been doing this a medium amount, 6.01 to $8.00=$ have been doing this a lot. The higher score indicates greater coping by the respondents. It is a validated instrument in which the Cronbach's alpha values range $0.50-0.90$, with only 3 coping strategies falling below $0.60^{21}$.

Table 1

Items of Depression, Anxiety and Stress Scale-21 with their respective.

\begin{tabular}{|llll|}
\hline & Depression & Anxiety & Stress \\
\hline Normal & $0-9$ & $0-7$ & $0-14$ \\
Mild & $10-13$ & $8-9$ & $15-18$ \\
Moderate & $14-20$ & $10-14$ & $19-25$ \\
Severe & $21-27$ & $15-19$ & $26-33$ \\
Extremely Severe & $28+$ & $20+$ & 34 \\
\hline
\end{tabular}

The questionnaire was pretested on 13 randomly selected undergraduate medical students of Hawasa University. After checking the collected data for completeness, it was double entered into Epi-data version 3.1 and exported into SPSS version 21 for analysis. Incomplete and inconsistent data were excluded from 
the analysis. The data were processed by using descriptive analysis, including frequency distribution, cross tabulation and summary measures. Bivariate logistic regression was used to measure the association between independent variables with dependent variables. Multivariate logistic regression analysis was carried out to find the role of each significant variable in determining the relevant sub-scale scores. Statistical significance was accepted at $p<0.05$.

\section{Results}

\section{Sociodemographic Characteristics}

From a total of 265 medical students who received the questionnaire, 260 completed the survey, yielding an overall response rate of $98.1 \%$. Age of the study sample ranged between 18 and 27 years with the mean (SD) of $22.03( \pm 2.62)$ years. In the present study, most respondents were male $(63.1 \%)$, single in marital status (75.4\%), living in campus (91.5\%) and had monthly income of $\leq 700$ ETB (52.7\%)(Table 2). Regarding their academic year, religion and ethnicity, 54 (20.8\%) were from the sixth year, 130 (50\%) were Orthodox believers and 139 (53.9\%) were Oromo respectively. 
Table 2

Sociodemographic characteristics of study participants in relation to gender, $N=260, A U$, January 2019.

\begin{tabular}{|c|c|c|c|c|}
\hline \multicolumn{2}{|c|}{ Sociodemographic Variables } & \multirow{2}{*}{$\begin{array}{l}\text { Male } \\
36(54.5 \%)\end{array}$} & \multirow{2}{*}{$\begin{array}{l}\text { Female } \\
30(45.5 \%)\end{array}$} & \multirow{2}{*}{$\begin{array}{l}\text { Total }(\mathrm{N}=260) \\
66(100.0 \%)\end{array}$} \\
\hline Age & $<20$ Years & & & \\
\hline & 20-24 Years & $91(67.4 \%)$ & $44(32.6 \%)$ & $135(100.0 \%)$ \\
\hline & $>24$ Years & $37(62.7 \%)$ & $22(37.3 \%)$ & $59(100.0 \%)$ \\
\hline \multirow[t]{2}{*}{ Marital Status } & Single & $128(65.3 \%)$ & $68(34.7 \%)$ & $196(100.0 \%)$ \\
\hline & Married & $36(56.3 \%)$ & $28(43.8 \%)$ & $64(100.0 \%)$ \\
\hline \multirow[t]{2}{*}{ Monthly Income } & $\leq 700 \mathrm{ETB}$ & $87(63.5 \%)$ & $50(36.5 \%)$ & $137(100.0 \%)$ \\
\hline & $>700$ ETB & $77(62.6 \%)$ & $46(37.4 \%)$ & $123(100.0 \%)$ \\
\hline \multirow[t]{6}{*}{ Educational Year } & 1st Year & $27(61.4 \%)$ & $17(38.6 \%)$ & $44(100.0 \%)$ \\
\hline & 2nd Year & $22(48.9 \%)$ & $23(51.1 \%)$ & $45(100.0 \%)$ \\
\hline & 3rd Year & $23(59.0 \%)$ & $16(41.0 \%)$ & $39(100.0 \%)$ \\
\hline & 4th Year & $36(76.6 \%)$ & $11(23.4 \%)$ & $47(100.0 \%)$ \\
\hline & 5th Year & $23(74.2 \%)$ & $8(25.8 \%)$ & $31(100.0 \%)$ \\
\hline & 6th Year & $33(61.1 \%)$ & $21(38.9 \%)$ & $54(100.0 \%)$ \\
\hline \multirow[t]{2}{*}{ Residency } & Non-dormitory & $11(50.0 \%)$ & $11(50.0 \%)$ & $22(100.0 \%)$ \\
\hline & Dormitory & $153(64.3 \%)$ & $85(35.7 \%)$ & $238(100.0 \%)$ \\
\hline \multirow[t]{4}{*}{ Religion } & Orthodox & $80(61.5 \%)$ & $50(38.5 \%)$ & $130(100.0 \%)$ \\
\hline & Muslim & $40(62.5 \%)$ & $24(37.5 \%)$ & $64(100.0 \%)$ \\
\hline & Protestant & $42(67.7 \%)$ & $20(32.3 \%)$ & $62(100.0 \%)$ \\
\hline & Others ${ }^{¥}$ & $2(50.0 \%)$ & $2(50.0 \%)$ & $4(100.0 \%)$ \\
\hline \multirow[t]{6}{*}{ Ethnicity } & Oromo & 97 (69.8\%) & $42(30.2 \%)$ & 139 (100.0\%) \\
\hline & Amhara & 38 (53.5\%) & $33(46.5 \%)$ & $71(100.0 \%)$ \\
\hline & Sidama & $2(33.3 \%)$ & $4(66.7 \%)$ & $6(100.0 \%)$ \\
\hline & Tigre & $7(50.0 \%)$ & $7(50.0 \%)$ & 14 (100.0\%) \\
\hline & Wolayita & $5(62.5 \%)$ & $3(37.5 \%)$ & $8(100.0 \%)$ \\
\hline & Gurage & $12(66.7 \%)$ & $6(33.3 \%)$ & 18 (100.0\%) \\
\hline
\end{tabular}

Key: - ${ }^{\ddagger}$ Catholic, Waqefatta, Faith and Pagan. ${ }^{\dagger+}$ Silte, Harari and Kambata. ETB = Ethiopian Birr. Current exchange rate: $\$ 1$ USD $=40.05$ ETB. 


\begin{tabular}{|cccc|}
\hline Sociodemographic Variables & Male & Female & Total $(\mathbf{N}=\mathbf{2 6 0})$ \\
\hline Others $^{\dagger \dagger}$ & $3(75.0 \%)$ & $1(25.0 \%)$ & $4(100.0 \%)$ \\
\hline $\begin{array}{l}\text { Key: }{ }^{+}{ }^{*} \text { Catholic, Waqefatta, Faith and Pagan. } \\
\text { Current exchange rate: } \$ \text { \$ } 1 \text { USD }=40.05 \text { ETB. }\end{array}$ & & & \\
\hline
\end{tabular}

\section{Prevalence of Depression, Anxiety and Stress}

The present study demonstrated a strong internal consistency between depression and anxiety $(r=0.494$, $p<0.001)$, depression and stress $(r=0.456, p<0.001)$, stress and anxiety $(r=0.420, p<0.001)$. From 260 valid analyzed questionnaires, the proportion of respondents detected to have depression, anxiety, and stress symptoms were $52.3 \%, 60.8 \%$, and $40.4 \%$ respectively. Whereas, the percentage of respondents who had extremely severe symptoms of depression, anxiety and stress were $6.2 \%, 16.2 \%$ and $2.3 \%$ respectively (Table 3). 
Table 3

Severity distribution of depression, anxiety and stress among study participants in relation to gender, $\mathrm{N}=260, \mathrm{AU}$, January 2019 .

\begin{tabular}{|c|c|c|c|c|}
\hline \multirow[t]{3}{*}{ Sub-scale } & & \multicolumn{2}{|l|}{ Sex } & \multirow{3}{*}{$\begin{array}{l}\text { Total } \\
\mathrm{N}(\%)\end{array}$} \\
\hline & & \multirow{2}{*}{$\begin{array}{l}\text { Male } \\
\mathrm{N}(\%)\end{array}$} & \multirow{2}{*}{$\begin{array}{l}\text { Female } \\
\mathrm{N}(\%)\end{array}$} & \\
\hline & & & & \\
\hline \multirow[t]{5}{*}{ Depression } & Normal & $85(50.6)$ & $39(42.4)$ & $124(47.7)$ \\
\hline & Mild & $34(20.2)$ & $20(21.7)$ & $54(20.8)$ \\
\hline & Moderate & $29(17.3)$ & $16(17.4)$ & $45(17.3)$ \\
\hline & Severe & $15(8.9)$ & $6(6.5)$ & $21(8.1)$ \\
\hline & Extremely Severe & $5(3.0)$ & $11(12.0)$ & $16(6.2)$ \\
\hline \multirow[t]{5}{*}{ Anxiety } & Normal & $73(43.5)$ & $29(31.5)$ & $102(39.2)$ \\
\hline & Mild & $12(7.1)$ & $10(10.9)$ & $22(8.5)$ \\
\hline & Moderate & $44(26.2)$ & $19(20.7)$ & $63(24.2)$ \\
\hline & Severe & $20(11.9)$ & $11(12.0)$ & $31(11.9)$ \\
\hline & Extremely Severe & 19 (11.3) & $23(25.0)$ & $42(16.2)$ \\
\hline \multirow[t]{5}{*}{ Stress } & Normal & $104(61.9)$ & $51(55.4)$ & 155 (59.6) \\
\hline & Mild & $23(13.7)$ & $14(15.2)$ & $37(14.2)$ \\
\hline & Moderate & $31(18.5)$ & $10(10.9)$ & $41(15.8)$ \\
\hline & Severe & $8(4.8 \%)$ & $13(14.1 \%)$ & $21(8.1 \%)$ \\
\hline & Extremely Severe & $2(1.2 \%)$ & $4(4.3 \%)$ & $6(2.3 \%)$ \\
\hline
\end{tabular}

\section{Association of Sociodemographic Characteristics with Depression}

A binary logistic regression analysis was applied to evaluate the possible impact of sociodemographic characteristics of respondents' depression level (Table 4). In the present study, depression had no statistical association with age, educational year, religion and ethnicity. Conversely, it had a statistically significant association with respondents' gender, in which males were 0.59 times less likely to be depressed than females (95\% Cl: 0.36-0.99). It was also associated with a monthly income, in which respondents who had monthly income of $\leq 700$ ETB were 1.68 times more depressed than above 700 ETB (95\% Cl: 1.03-2.74). Depression was also associated with marital status, in which respondents who hadn't relationship (single) were 0.53 times less likely depressed than those in a relationship (married) (95\%: 0.30-0.95). Finally, it was also statistically associated with residency, in which non-dormitory living respondents were 10.52 more likely depressed than those living in dormitory (95\% Cl: $2.41-46.00)$. 
Table 4

Logistic regression of sociodemographic risk factors versus depression, $N=260, A U$, January 2019.

\begin{tabular}{|c|c|c|c|c|c|c|}
\hline \multicolumn{2}{|c|}{ Socio-demographic variables } & \multirow{3}{*}{$\begin{array}{l}\text { Total } \\
\text { N (\%) }\end{array}$} & \multicolumn{2}{|c|}{ Depression } & \multirow[t]{3}{*}{ COR } & \multirow[t]{3}{*}{ AOR } \\
\hline & & & Yes & No & & \\
\hline & & & $\mathrm{N}(\%)$ & $\mathrm{N}(\%)$ & & \\
\hline \multirow[t]{3}{*}{ Age } & $<20$ years & $\begin{array}{l}66 \\
(100.0)\end{array}$ & $\begin{array}{l}35 \\
(53.0)\end{array}$ & $\begin{array}{l}31 \\
(47.0)\end{array}$ & $\begin{array}{l}1.09(0.54- \\
2.20)\end{array}$ & \\
\hline & $20-24$ years & $\begin{array}{l}135 \\
(100.0)\end{array}$ & $\begin{array}{l}71 \\
(52.6)\end{array}$ & $\begin{array}{l}64 \\
(47.4)\end{array}$ & $\begin{array}{l}1.07(0.58- \\
1.98)\end{array}$ & \\
\hline & $>24$ years & $\begin{array}{l}59 \\
(100.0)\end{array}$ & $\begin{array}{l}30 \\
(50.8)\end{array}$ & $\begin{array}{l}29 \\
(49.2)\end{array}$ & $1.00 *$ & \\
\hline \multirow[t]{2}{*}{ Sex } & Male & $\begin{array}{l}164 \\
(100.0)\end{array}$ & $\begin{array}{l}78 \\
(47.6)\end{array}$ & $\begin{array}{l}86 \\
(52.4)\end{array}$ & $\begin{array}{l}0.59(0.36- \\
0.99)\end{array}$ & $\begin{array}{l}0.63(0.37- \\
1.08)\end{array}$ \\
\hline & Female & $\begin{array}{l}96 \\
(100.0)\end{array}$ & $\begin{array}{l}58 \\
(60.4)\end{array}$ & $\begin{array}{l}38 \\
(39.6)\end{array}$ & $1.00 *$ & $1.00 *$ \\
\hline \multirow[t]{2}{*}{$\begin{array}{l}\text { Monthly } \\
\text { income }\end{array}$} & $\leq 700$ ETB & $\begin{array}{l}137 \\
(100.0)\end{array}$ & $\begin{array}{l}80 \\
(58.4)\end{array}$ & $\begin{array}{l}57 \\
(41.6)\end{array}$ & $\begin{array}{l}1.68(1.03- \\
2.74)\end{array}$ & $\begin{array}{l}1.97(1.17- \\
3.33)\end{array}$ \\
\hline & $>700$ ETB & $\begin{array}{l}123 \\
(100.0)\end{array}$ & $\begin{array}{l}56 \\
(45.5)\end{array}$ & $\begin{array}{l}67 \\
(54.5)\end{array}$ & $1.00 *$ & $1.00 *$ \\
\hline \multirow[t]{2}{*}{$\begin{array}{l}\text { Marital } \\
\text { status }\end{array}$} & Single & $\begin{array}{l}196 \\
(100.0)\end{array}$ & $\begin{array}{l}95 \\
(48.5)\end{array}$ & $\begin{array}{l}101 \\
(51.5)\end{array}$ & $\begin{array}{l}0.53(0.30- \\
0.95)\end{array}$ & $\begin{array}{l}0.60(0.33- \\
1.11)\end{array}$ \\
\hline & Married & $\begin{array}{l}64 \\
(100.0)\end{array}$ & $\begin{array}{l}41 \\
(64.1)\end{array}$ & $\begin{array}{l}23 \\
(35.9)\end{array}$ & $1.00 *$ & $1.00^{*}$ \\
\hline \multirow[t]{6}{*}{$\begin{array}{l}\text { Educational } \\
\text { year }\end{array}$} & First year & $\begin{array}{l}44 \\
(100.0)\end{array}$ & $\begin{array}{l}23 \\
(52.3)\end{array}$ & $\begin{array}{l}21 \\
(47.7)\end{array}$ & $\begin{array}{l}1.27(0.57- \\
2.82)\end{array}$ & \\
\hline & Second year & $\begin{array}{l}45 \\
(100.0)\end{array}$ & $\begin{array}{l}26 \\
(57.8)\end{array}$ & $\begin{array}{l}19 \\
(42.2)\end{array}$ & $\begin{array}{l}1.59(0.72- \\
3.52)\end{array}$ & \\
\hline & Third year & $\begin{array}{l}39 \\
(100.0)\end{array}$ & $\begin{array}{l}18 \\
(46.2)\end{array}$ & $\begin{array}{l}21 \\
(53.8)\end{array}$ & $\begin{array}{l}0.99(0.44- \\
2.27)\end{array}$ & \\
\hline & Fourth year & $\begin{array}{l}47 \\
(100.0)\end{array}$ & $\begin{array}{l}25 \\
(53.2)\end{array}$ & $\begin{array}{l}22 \\
(46.8)\end{array}$ & $\begin{array}{l}1.32(0.60- \\
2.89)\end{array}$ & \\
\hline & Fifth year & $\begin{array}{l}31 \\
(100.0)\end{array}$ & $\begin{array}{l}19 \\
(61.3)\end{array}$ & $\begin{array}{l}12 \\
(38.7)\end{array}$ & $\begin{array}{l}1.84(0.75- \\
4.51)\end{array}$ & \\
\hline & Sixth year & $\begin{array}{l}54 \\
(100.0)\end{array}$ & $\begin{array}{l}25 \\
(46.3)\end{array}$ & $\begin{array}{l}29 \\
(53.7)\end{array}$ & $1.00 *$ & \\
\hline Residency & Nondormitory & $\begin{array}{l}22 \\
(100.0)\end{array}$ & $\begin{array}{l}20 \\
(90.9)\end{array}$ & $2(9.1)$ & $\begin{array}{l}10.52(2.41- \\
46.0)\end{array}$ & $\begin{array}{l}12.55(2.79- \\
56.37)\end{array}$ \\
\hline
\end{tabular}

Key: - *Reference category. 


\begin{tabular}{|c|c|c|c|c|c|c|}
\hline & Dormitory & $\begin{array}{l}238 \\
(100.0)\end{array}$ & $\begin{array}{l}116 \\
(48.7)\end{array}$ & $\begin{array}{l}122 \\
(51.3)\end{array}$ & $1.00 *$ & $1.00 *$ \\
\hline \multirow[t]{4}{*}{ Religion } & Orthodox & $\begin{array}{l}130 \\
(100.0)\end{array}$ & $\begin{array}{l}68 \\
(52.3)\end{array}$ & $\begin{array}{l}62 \\
(47.7)\end{array}$ & $\begin{array}{l}4.39(0.43- \\
44.51)\end{array}$ & \\
\hline & Muslim & $\begin{array}{l}64 \\
(100.0)\end{array}$ & $\begin{array}{l}38 \\
(59.4)\end{array}$ & $\begin{array}{l}26 \\
(40.6)\end{array}$ & $\begin{array}{l}3.29(0.33- \\
32.47)\end{array}$ & \\
\hline & Protestant & $\begin{array}{l}62 \\
(100.0)\end{array}$ & $\begin{array}{l}29 \\
(46.8)\end{array}$ & $\begin{array}{l}33 \\
(53.2)\end{array}$ & $\begin{array}{l}2.64(0.26- \\
26.76)\end{array}$ & \\
\hline & Others & $\begin{array}{l}4 \\
(100.0)\end{array}$ & $1(25.0)$ & $3(75.0)$ & $1.00 *$ & \\
\hline \multirow[t]{7}{*}{ Ethnicity } & Oromo & $\begin{array}{l}139 \\
(100.0)\end{array}$ & $\begin{array}{l}67 \\
(48.2)\end{array}$ & $\begin{array}{l}72 \\
(51.8)\end{array}$ & $\begin{array}{l}0.93(0.13- \\
6.79)\end{array}$ & \\
\hline & Amhara & $\begin{array}{l}71 \\
(100.0)\end{array}$ & $\begin{array}{l}40 \\
(56.3)\end{array}$ & $\begin{array}{l}31 \\
(43.7)\end{array}$ & $\begin{array}{l}1.29(0.17- \\
9.68)\end{array}$ & \\
\hline & Sidama & $\begin{array}{l}6 \\
(100.0)\end{array}$ & $3(50.0)$ & $3(50.0)$ & $\begin{array}{l}1.00(0.08- \\
12.56)\end{array}$ & \\
\hline & Tigre & $\begin{array}{l}14 \\
(100.0)\end{array}$ & $7(50.0)$ & $7(50.0)$ & $\begin{array}{l}1.00(0.11- \\
9.23)\end{array}$ & \\
\hline & Wolayita & $\begin{array}{l}8 \\
(100.0)\end{array}$ & $6(75.0)$ & $2(25.0)$ & $\begin{array}{l}3.00(0.24- \\
37.67)\end{array}$ & \\
\hline & Gurage & $\begin{array}{l}18 \\
(100.0)\end{array}$ & $\begin{array}{l}11 \\
(61.1)\end{array}$ & 7 (38.9) & $\begin{array}{l}1.57(0.18- \\
13.86)\end{array}$ & \\
\hline & Others & $\begin{array}{l}4 \\
(100.0)\end{array}$ & $2(50.0)$ & $2(50.0)$ & $1.00 *$ & \\
\hline
\end{tabular}

Key: - *Reference category.

\section{Association of Sociodemographic Characteristics with Anxiety}

In a binary logistic regression analysis of the present study, anxiety had no significant statistical association with sociodemographic variables like monthly income, religion and ethnicity (Table 5). Contrariwise, it was statistically associated with the age of respondents, with individuals in the age range between 20-24 being 1.92 times less likely to be anxious than respondents $>24$ years ( $95 \% \mathrm{Cl}$ : $0.34-$ 0.99). It was also associated with the gender of respondents; males were 0.58 less likely to be anxious as compared with females (95\% Cl: 0.34-0.99). Anxiety was also associated with marital status, respondents who were not in a relationship (single) were 0.52 times less likely to be anxious than those in a relationship (married)(95\%: 0.28-0.96). Concerning its association with the educational level of respondents, third-year respondents were 4.85 more likely to be anxious than sixth-year students (95\% Cl: 1.93-12.19). Finally, anxiety was also associated with residency, non-dormitory living respondents were 15.48 more likely to be anxious than those living in a dormitory (95\% Cl: 2.05-117.00). 
Table 5

Logistic regression of socio-demographic risk factors versus anxiety, N =260, AU, January 2019.

\begin{tabular}{|c|c|c|c|c|c|c|}
\hline \multicolumn{2}{|c|}{ Sociodemographic variables } & \multirow{3}{*}{$\begin{array}{l}\text { Total } \\
\mathrm{N}=260\end{array}$} & \multicolumn{2}{|c|}{ Anxiety } & \multirow[t]{3}{*}{ COR } & \multirow[t]{3}{*}{ AOR } \\
\hline & & & Yes & No & & \\
\hline & & & $\mathrm{N}(\%)$ & $\mathrm{N}(\%)$ & & \\
\hline \multirow[t]{3}{*}{ Age } & $<20$ years & $\begin{array}{l}66 \\
(100.0)\end{array}$ & $\begin{array}{l}41 \\
(62.1)\end{array}$ & $\begin{array}{l}25 \\
(37.9)\end{array}$ & $\begin{array}{l}1.70(0.83- \\
3.46)\end{array}$ & $\begin{array}{l}0.41(0.09- \\
1.88)\end{array}$ \\
\hline & $20-24$ years & $\begin{array}{l}135 \\
(100.0)\end{array}$ & $\begin{array}{l}88 \\
(65.2)\end{array}$ & $\begin{array}{l}47 \\
(34.8)\end{array}$ & $\begin{array}{l}1.94(1.04- \\
3.61)\end{array}$ & $\begin{array}{l}0.64(0.24- \\
1.69)\end{array}$ \\
\hline & $>24$ years & $\begin{array}{l}59 \\
(100.0)\end{array}$ & $\begin{array}{l}29 \\
(49.2)\end{array}$ & $\begin{array}{l}30 \\
(50.8)\end{array}$ & $1.00 \star$ & $1.00 *$ \\
\hline \multirow[t]{2}{*}{ Sex } & Male & $\begin{array}{l}164 \\
(100.0)\end{array}$ & $\begin{array}{l}92 \\
(56.1)\end{array}$ & $\begin{array}{l}72 \\
(43.9)\end{array}$ & $\begin{array}{l}0.58(0.34- \\
0.99)\end{array}$ & $\begin{array}{l}0.60(0.33- \\
1.08)\end{array}$ \\
\hline & Female & $\begin{array}{l}96 \\
(100.0)\end{array}$ & $\begin{array}{l}66 \\
(68.8)\end{array}$ & $\begin{array}{l}30 \\
(31.3)\end{array}$ & $1.00 *$ & $1.00 *$ \\
\hline \multirow[t]{2}{*}{$\begin{array}{l}\text { Monthly } \\
\text { income }\end{array}$} & $\leq 700 \mathrm{ETB}$ & $\begin{array}{l}137 \\
(100.0)\end{array}$ & $\begin{array}{l}89 \\
(65.0)\end{array}$ & $\begin{array}{l}48 \\
(35.0)\end{array}$ & $\begin{array}{l}1.45(0.88- \\
2.39)\end{array}$ & \\
\hline & $>700$ ETB & $\begin{array}{l}123 \\
(100.0)\end{array}$ & $\begin{array}{l}69 \\
(56.1)\end{array}$ & $\begin{array}{l}54 \\
(43.9)\end{array}$ & $1.00 *$ & \\
\hline \multirow[t]{2}{*}{$\begin{array}{l}\text { Marital } \\
\text { status }\end{array}$} & Single & $\begin{array}{l}196 \\
(100.0)\end{array}$ & $\begin{array}{l}112 \\
(57.1)\end{array}$ & $\begin{array}{l}84 \\
(42.9)\end{array}$ & $\begin{array}{l}0.52(0.28- \\
0.96)\end{array}$ & $\begin{array}{l}0.52(0.26- \\
1.03)\end{array}$ \\
\hline & Married & $\begin{array}{l}64 \\
(100.0)\end{array}$ & $\begin{array}{l}46 \\
(71.9)\end{array}$ & $\begin{array}{l}18 \\
(28.1)\end{array}$ & $1.00 *$ & $1.00 *$ \\
\hline \multirow[t]{6}{*}{$\begin{array}{l}\text { Educational } \\
\text { year }\end{array}$} & First year & $\begin{array}{l}44 \\
(100.0)\end{array}$ & $\begin{array}{l}25 \\
(56.8)\end{array}$ & $\begin{array}{l}19 \\
(43.2)\end{array}$ & $\begin{array}{l}1.91(0.85- \\
4.28)\end{array}$ & $\begin{array}{l}8.31(1.65- \\
41.77)\end{array}$ \\
\hline & Second year & $\begin{array}{l}45 \\
(100.0)\end{array}$ & $\begin{array}{l}34 \\
(75.6)\end{array}$ & $\begin{array}{l}11 \\
(24.4)\end{array}$ & $\begin{array}{l}4.50(1.88- \\
10.73)^{-}\end{array}$ & $\begin{array}{l}14.89 \\
65.23)\end{array}$ \\
\hline & Third year & $\begin{array}{l}39 \\
(100.0)\end{array}$ & $\begin{array}{l}30 \\
(76.9)\end{array}$ & $\begin{array}{l}9 \\
(23.1)\end{array}$ & $\begin{array}{l}4.85(1.93- \\
12.19)\end{array}$ & $\begin{array}{l}12.78(3.45- \\
47.37)\end{array}$ \\
\hline & Fourth year & $\begin{array}{l}47 \\
(100.0)\end{array}$ & $\begin{array}{l}29 \\
(61.7)\end{array}$ & $\begin{array}{l}18 \\
(38.3)\end{array}$ & $\begin{array}{l}2.34(1.05- \\
5.22)\end{array}$ & $\begin{array}{l}6.54(2.02- \\
21.16)\end{array}$ \\
\hline & Fifth year & $\begin{array}{l}31 \\
(100.0)\end{array}$ & $\begin{array}{l}18 \\
(58.1)\end{array}$ & $\begin{array}{l}13 \\
(41.9)\end{array}$ & $\begin{array}{l}2.014(0.82- \\
4.94)\end{array}$ & $\begin{array}{l}5.47(1.78- \\
16.84)\end{array}$ \\
\hline & Sixth year & $\begin{array}{l}54 \\
(100.0)\end{array}$ & $\begin{array}{l}22 \\
(40.7)\end{array}$ & $\begin{array}{l}32 \\
(59.3)\end{array}$ & $1.00 *$ & $1.00 *$ \\
\hline Residency & Nondormitory & $\begin{array}{l}22 \\
(100.0)\end{array}$ & $\begin{array}{l}21 \\
(95.5)\end{array}$ & $1(4.5)$ & $\begin{array}{l}15.48(2.05- \\
116.0)\end{array}$ & $\begin{array}{l}42.11(4.88- \\
363.36)\end{array}$ \\
\hline
\end{tabular}

Key: - *Reference category. 


\begin{tabular}{|c|c|c|c|c|c|c|}
\hline & Dormitory & $\begin{array}{l}238 \\
(100.0)\end{array}$ & $\begin{array}{l}137 \\
(57.6)\end{array}$ & $\begin{array}{l}101 \\
(42.4)\end{array}$ & $1.00 *$ & $1.00 *$ \\
\hline \multirow[t]{4}{*}{ Religion } & Orthodox & $\begin{array}{l}130 \\
(100.0)\end{array}$ & $\begin{array}{l}75 \\
(57.7)\end{array}$ & $\begin{array}{l}55 \\
(42.3)\end{array}$ & $\begin{array}{l}0.93(0.40- \\
2.17)\end{array}$ & \\
\hline & Muslim & $\begin{array}{l}64 \\
(100.0)\end{array}$ & $\begin{array}{l}45 \\
(70.3)\end{array}$ & $\begin{array}{l}19 \\
(29.7)\end{array}$ & $\begin{array}{l}0.79(0.31- \\
2.03)\end{array}$ & \\
\hline & Protestant & $\begin{array}{l}62 \\
(100.0)\end{array}$ & $\begin{array}{l}34 \\
(54.8)\end{array}$ & $\begin{array}{l}28 \\
(45.2)\end{array}$ & $\begin{array}{l}0.77(0.31- \\
1.90)\end{array}$ & \\
\hline & Others & $\begin{array}{l}4 \\
(100.0)\end{array}$ & $\begin{array}{l}4 \\
(100.0)\end{array}$ & $0(0.0)$ & $1.00 *$ & \\
\hline \multirow[t]{7}{*}{ Ethnicity } & Oromo & $\begin{array}{l}139 \\
(100.0)\end{array}$ & $\begin{array}{l}82 \\
(59.0)\end{array}$ & $\begin{array}{l}57 \\
(41.0)\end{array}$ & $\begin{array}{l}1.44(0.20- \\
10.51)\end{array}$ & \\
\hline & Amhara & $\begin{array}{l}71 \\
(100.0)\end{array}$ & $\begin{array}{l}42 \\
(59.2)\end{array}$ & $\begin{array}{l}29 \\
(40.8)\end{array}$ & $\begin{array}{l}1.45(0.19- \\
10.88)\end{array}$ & \\
\hline & Sidama & $\begin{array}{l}6 \\
(100.0)\end{array}$ & $\begin{array}{l}5 \\
(83.3)\end{array}$ & $\begin{array}{l}1 \\
(16.7)\end{array}$ & $\begin{array}{l}5.00(0.27- \\
91.52)\end{array}$ & \\
\hline & Tigre & $\begin{array}{l}14 \\
(100.0)\end{array}$ & $\begin{array}{l}10 \\
(71.4)\end{array}$ & $\begin{array}{l}4 \\
(28.6)\end{array}$ & $\begin{array}{l}2.50(0.26- \\
24.38)\end{array}$ & \\
\hline & Wolayita & $\begin{array}{l}8 \\
(100.0)\end{array}$ & $\begin{array}{l}5 \\
(62.5)\end{array}$ & $\begin{array}{l}3 \\
(37.5)\end{array}$ & $\begin{array}{l}1.67(0.15- \\
18.87)\end{array}$ & \\
\hline & Gurage & $\begin{array}{l}18 \\
(100.0)\end{array}$ & $\begin{array}{l}12 \\
(66.7)\end{array}$ & $\begin{array}{l}6 \\
(33.3)\end{array}$ & $\begin{array}{l}2.00(0.22- \\
17.89)\end{array}$ & \\
\hline & Others & $\begin{array}{l}4 \\
(100.0)\end{array}$ & $\begin{array}{l}2 \\
(50.0)\end{array}$ & $\begin{array}{l}2 \\
(50.0)\end{array}$ & $1.00 *$ & \\
\hline
\end{tabular}

Key: - *Reference category.

\section{Association of Sociodemographic Characteristics with Stress}

In the present study, when the impact of sociodemographic characteristics on the stress level was evaluated by binary logistic regression analysis, stress had no association with religion and ethnicity (Table 6). But it had a significant statistical association with the age of respondents, with respondents younger than 20 years being 2.07 more likely stressed than those older than 24 years ( $95 \% \mathrm{Cl}$ : $1.01-$ 4.27). It was also associated with sex of respondents with males were 0.57 times less likely stressed than females (95\% Cl: 0.34-0.95). Statistically, it was also associated to monthly income, participants who had a monthly income of $\leq 700$ ETB were 1.87 times more likely stressed than those respondents who had monthly income of above 700 ETB (95\% Cl: 1.13-3.10). Stress was also associated with marital status, respondents who hadn't relationship (single) were 0.55 times less likely stressed than those in a relationship (married)(95\%: 0.31-0.96). 
Concerning to its association with the educational level of respondents, second year respondents were, 4.92 times more likely stressed than those in sixth year (95\% Cl: 2.09-11.61). Finally, it is also statistically associated with residency, non-dormitory living respondents were 3.52 more likely stressed than those in dormitory (95\% Cl: $1.38-8.97)$. 
Table 6

Logistic regression of socio-demographic risk factors versus stress, $N=260, A U$, January 2019.

\begin{tabular}{|c|c|c|c|c|c|c|}
\hline \multicolumn{2}{|c|}{ Socio-demographic variables } & \multirow{3}{*}{$\begin{array}{l}\text { Total } \\
\mathrm{N}=260\end{array}$} & \multicolumn{2}{|l|}{ Stress } & \multirow[t]{3}{*}{ COR } & \multirow[t]{3}{*}{ AOR } \\
\hline & & & Yes & No & & \\
\hline & & & $\mathrm{N}(\%)$ & $\mathrm{N}(\%)$ & & \\
\hline \multirow[t]{3}{*}{ Age } & $<20$ years & $\begin{array}{l}66 \\
(100.0)\end{array}$ & $\begin{array}{l}34 \\
(51.5)\end{array}$ & $\begin{array}{l}32 \\
(48.5)\end{array}$ & $\begin{array}{l}2.07(1.01- \\
4.27)\end{array}$ & $\begin{array}{l}2.08(0.47- \\
9.31)\end{array}$ \\
\hline & $20-24$ years & $\begin{array}{l}135 \\
(100.0)\end{array}$ & $\begin{array}{l}51 \\
(37.8)\end{array}$ & $\begin{array}{l}84 \\
(62.2)\end{array}$ & $\begin{array}{l}1.18(0.62- \\
2.25)\end{array}$ & $\begin{array}{l}0.98(0.39- \\
2.47)\end{array}$ \\
\hline & $>24$ years & $\begin{array}{l}59 \\
(100.0)\end{array}$ & $\begin{array}{l}20 \\
(33.9)\end{array}$ & $\begin{array}{l}39 \\
(66.1)\end{array}$ & $1.00 *$ & $1.00 *$ \\
\hline \multirow[t]{2}{*}{ Sex } & Male & $\begin{array}{l}164 \\
(100.0)\end{array}$ & $\begin{array}{l}58 \\
(35.4)\end{array}$ & $\begin{array}{l}106 \\
(64.6)\end{array}$ & $\begin{array}{l}0.57(0.34- \\
0.95)\end{array}$ & $\begin{array}{l}0.66(0.37- \\
1.16)\end{array}$ \\
\hline & Female & $\begin{array}{l}96 \\
(100.0)\end{array}$ & $\begin{array}{l}47 \\
(49.0)\end{array}$ & $\begin{array}{l}49 \\
(51.0)\end{array}$ & $1.00 *$ & $1.00 *$ \\
\hline \multirow[t]{2}{*}{$\begin{array}{l}\text { Monthly } \\
\text { income }\end{array}$} & $\leq 700$ ETB & $\begin{array}{l}137 \\
(100.0)\end{array}$ & $\begin{array}{l}65 \\
(47.4)\end{array}$ & $\begin{array}{l}72 \\
(52.6)\end{array}$ & $\begin{array}{l}1.87(1.13- \\
3.10)\end{array}$ & $\begin{array}{l}1.99(1.00- \\
3.98)\end{array}$ \\
\hline & $>700$ ETB & $\begin{array}{l}123 \\
(100.0)\end{array}$ & $\begin{array}{l}40 \\
(32.5)\end{array}$ & $\begin{array}{l}83 \\
(67.5)\end{array}$ & $1.00 *$ & $1.00 *$ \\
\hline \multirow[t]{2}{*}{ Marital status } & Single & $\begin{array}{l}196 \\
(100.0)\end{array}$ & $\begin{array}{l}72 \\
(36.7)\end{array}$ & $\begin{array}{l}124 \\
(63.3)\end{array}$ & $\begin{array}{l}0.55(0.31- \\
0.96)\end{array}$ & $\begin{array}{l}0.56(0.30- \\
1.05)\end{array}$ \\
\hline & Married & $\begin{array}{l}64 \\
(100.0)\end{array}$ & $\begin{array}{l}33 \\
(51.6)\end{array}$ & $\begin{array}{l}31 \\
(48.4)\end{array}$ & $1.00 *$ & $1.00 *$ \\
\hline \multirow[t]{6}{*}{$\begin{array}{l}\text { Educational } \\
\text { year }\end{array}$} & First year & $\begin{array}{l}44 \\
(100.0)\end{array}$ & $\begin{array}{l}15 \\
(34.1)\end{array}$ & $\begin{array}{l}29 \\
(65.9)\end{array}$ & $\begin{array}{l}1.03(0.45- \\
2.40)\end{array}$ & $\begin{array}{l}0.51(0.10- \\
2.63)\end{array}$ \\
\hline & Second year & $\begin{array}{l}45 \\
(100.0)\end{array}$ & $\begin{array}{l}32 \\
(71.1)\end{array}$ & $\begin{array}{l}13 \\
(28.9)\end{array}$ & $\begin{array}{l}4.92(2.09- \\
11.61)^{-}\end{array}$ & $\begin{array}{l}1.84(1.02- \\
11.21)^{-}\end{array}$ \\
\hline & Third year & $\begin{array}{l}39 \\
(100.0)\end{array}$ & $\begin{array}{l}10 \\
(25.6)\end{array}$ & $\begin{array}{l}29 \\
(74.4)\end{array}$ & $\begin{array}{l}0.69(0.28- \\
1.72)\end{array}$ & $\begin{array}{l}0.54(0.16- \\
1.86)\end{array}$ \\
\hline & Fourth year & $\begin{array}{l}47 \\
(100.0)\end{array}$ & $\begin{array}{l}18 \\
(38.3)\end{array}$ & $\begin{array}{l}29 \\
(61.7)\end{array}$ & $\begin{array}{l}1.24(0.55- \\
2.81)\end{array}$ & $\begin{array}{l}1.11(0.34- \\
3.57)\end{array}$ \\
\hline & Fifth year & $\begin{array}{l}31 \\
(100.0)\end{array}$ & $\begin{array}{l}12 \\
(38.7)\end{array}$ & $\begin{array}{l}19 \\
(61.3)\end{array}$ & $\begin{array}{l}1.26(0.50- \\
3.16)\end{array}$ & $\begin{array}{l}1.12(0.34- \\
3.69)\end{array}$ \\
\hline & Sixth year & $\begin{array}{l}54 \\
(100.0)\end{array}$ & $\begin{array}{l}18 \\
(33.3)\end{array}$ & $\begin{array}{l}36 \\
(66.7)\end{array}$ & $1.00 *$ & $1.00 *$ \\
\hline Residency & Nondormitory & $\begin{array}{l}22 \\
(100.0)\end{array}$ & $\begin{array}{l}15 \\
(68.2)\end{array}$ & 7 (31.8) & $\begin{array}{l}3.52(1.38- \\
8.97)\end{array}$ & $\begin{array}{l}4.93(1.73- \\
14.08)\end{array}$ \\
\hline
\end{tabular}

Key: - *Reference category. 


\begin{tabular}{|c|c|c|c|c|c|c|}
\hline & Dormitory & $\begin{array}{l}238 \\
(100.0)\end{array}$ & $\begin{array}{l}90 \\
(37.8)\end{array}$ & $\begin{array}{l}148 \\
(62.2)\end{array}$ & $1.00 *$ & $1.00 *$ \\
\hline \multirow[t]{4}{*}{ Religion } & Orthodox & $\begin{array}{l}130 \\
(100.0)\end{array}$ & $\begin{array}{l}50 \\
(38.5)\end{array}$ & $\begin{array}{l}80 \\
(61.5)\end{array}$ & $\begin{array}{l}0.83(0.11- \\
6.25)\end{array}$ & \\
\hline & Muslim & $\begin{array}{l}64 \\
(100.0)\end{array}$ & $\begin{array}{l}29 \\
(45.3)\end{array}$ & $\begin{array}{l}35 \\
(54.7)\end{array}$ & $\begin{array}{l}0.63(0.09- \\
4.58)\end{array}$ & \\
\hline & Protestant & $\begin{array}{l}62 \\
(100.0)\end{array}$ & $\begin{array}{l}24 \\
(38.7)\end{array}$ & $\begin{array}{l}38 \\
(61.3)\end{array}$ & $\begin{array}{l}0.63(0.08- \\
4.79)\end{array}$ & \\
\hline & Others & $4(100.0)$ & $\begin{array}{l}2 \\
(50.0)\end{array}$ & $2(50.0)$ & $1.00 *$ & \\
\hline \multirow[t]{7}{*}{ Ethnicity } & Oromo & $\begin{array}{l}139 \\
(100.0)\end{array}$ & $\begin{array}{l}55 \\
(39.6)\end{array}$ & $\begin{array}{l}84 \\
(60.4)\end{array}$ & $\begin{array}{l}0.22(0.02- \\
2.15)\end{array}$ & \\
\hline & Amhara & $\begin{array}{l}71 \\
(100.0)\end{array}$ & $\begin{array}{l}28 \\
(39.4)\end{array}$ & $\begin{array}{l}43 \\
(60.6)\end{array}$ & $\begin{array}{l}0.22(0.02- \\
2.19)\end{array}$ & \\
\hline & Sidama & $6(100.0)$ & $\begin{array}{l}4 \\
(66.7)\end{array}$ & 2 (33.3) & $\begin{array}{l}0.67(0.04- \\
11.29)\end{array}$ & \\
\hline & Tigre & $\begin{array}{l}14 \\
(100.0)\end{array}$ & $\begin{array}{l}6 \\
(42.9)\end{array}$ & $8(57.1)$ & $\begin{array}{l}0.25(0.02- \\
3.04)\end{array}$ & \\
\hline & Wolayita & $8(100.0)$ & $\begin{array}{l}2 \\
(25.0)\end{array}$ & $6(75.0)$ & $\begin{array}{l}0.11(0.01- \\
1.78)\end{array}$ & \\
\hline & Gurage & $\begin{array}{l}18 \\
(100.0)\end{array}$ & $\begin{array}{l}7 \\
(38.9)\end{array}$ & $\begin{array}{l}11 \\
(61.1)\end{array}$ & $\begin{array}{l}0.21(0.02- \\
2.47)\end{array}$ & \\
\hline & Others & $4(100.0)$ & $\begin{array}{l}3 \\
(75.0)\end{array}$ & $1(25.0)$ & $1.00 *$ & \\
\hline
\end{tabular}

Key: - *Reference category.

\section{Predictor Risk Factors For Depression}

From variables like gender, monthly income, marital status and residency who were candidates for multivariate logistic regressions ( $P$-value $<0.05)$, predictors of the depression were monthly income and residency (Table 7). In our study, the odd of being depressed was markedly increased with 1.97 times among respondents who had monthly income of $\leq 700$ ETB than $>700$ ETB (95\% Cl: 1.17-3.33). Similarly, the odds of being depressed, were markedly increased with 12.55 times among respondents living non-dormitory than living in dormitory (95\% Cl: 2.79-56.37).

\section{Predictor Risk Factors For Anxiety}

Age, sex, marital status, educational year and residency had $p<0.05$ that made them candidates for multivariate logistic regressions. Only educational year and residency were found to be predictors of the anxiety (Table 5). In the present study, the odds of being anxious were markedly decreased as educational level increased after the first year. For example, the odds of being anxious were markedly 
increased with 14.89 times in the second year students than sixth years (95\% Cl: 3.40-65.23). The odd of being anxious were also more likely increases with 42.11 times among participants living in nondormitory than in dormitory (95\% Cl: 4.88-363.36).

\section{Predictor Risk Factors For Stress}

In the present study, monthly income, educational year and residency were found to be predictors of the stress from all sociodemographic characteristics that were candidate for multivariate logistic regressions (Table 6). For instance, the odd of being stressed was markedly increased with 1.99 times among participants who had a monthly income of $\leq 700$ ETB than those who had above 700 ETB (95\% Cl: $1.00-$ 3.98). Similarly, the odds of being stressed were markedly increased with 1.84 times in second year, participants than sixth years (95\% Cl: 1.02-11.21). Furthermore, the odds of being stressed were more likely increases with 4.93 times among respondent living in non-dormitory than in dormitory $(95 \% \mathrm{Cl}$ : 1.73-14.08).

\section{Effectiveness of Coping Strategies}

The total coping strategies applied by respondents were 28 with the minimum score of 2 and maximum score of 8. Among coping strategies, "religious coping with mean ( \pm SD) coping score of 5.94 (1.85), active coping with 5.52 (1.75), positive reframing with 5.39 (1.67) and planning with 5.38 (1.71)" were found to be the axioms used by most respondents. Inversely, behavioral disengagement with mean ( \pm SD) coping score of 4.34 (1.75), denial with $4.10(1.70)$ and substance use with $3.47(1.81)$ " were found to be the least used coping strategies (Table 7). In the present study, the active coping strategies were the most commonly used strategies than avoidant strategies. However, there was a variation across gender concerning to specific types of coping strategies used by respondents. Females were more commonly involved in use of emotional support with mean ( \pm SD) coping score of 4.89 (1.67), use of instrumental support with 5.33 (1.82), positive reframing with 5.65 (1.77), humor with 4.93 (1.89), acceptance with 5.15 (1.78), religion with 6.09 (2.00) and self-blame with 4.78 (1.64) than males did. Contrariwise, males were more commonly used active coping with mean ( \pm SD) coping score of $5.56(1.71)$ and substance use with 3.49 (1.75) than females did (Table 7). 
Table 7

Rank of coping strategies according to mean score as rated by study participants in relation to gender, $\mathrm{N}=260, \mathrm{AU}$, January 2019.

\begin{tabular}{|c|c|c|c|}
\hline \multirow[t]{3}{*}{ Scale } & \multicolumn{2}{|l|}{ Sex } & \multirow{3}{*}{$\begin{array}{l}\text { Total }(\mathrm{N}=260) \\
\text { Mean }(\mathrm{SD})\end{array}$} \\
\hline & Male & Female & \\
\hline & Mean (SD) & Mean (SD) & \\
\hline Self-distraction & $5.09(1.41)$ & $5.06(1.69)$ & $5.08(1.52)$ \\
\hline Active coping* & $5.56(1.71)$ & $5.46(1.82)$ & $5.52(1.75)$ \\
\hline Denial & $4.12(1.65)$ & $4.07(1.80)$ & $4.10(1.70)$ \\
\hline Substance use* & $3.49(1.75)$ & $3.44(1.92)$ & $3.47(1.81)$ \\
\hline Use of emotional support** & 4.58 (1.59) & $4.89(1.67)$ & $4.69(1.63)$ \\
\hline Use of instrumental support** & $4.92(1.66)$ & $5.33(1.82)$ & $5.07(1.73)$ \\
\hline Behavioral disengagement & $4.33(1.73)$ & $4.37(1.81)$ & $4.34(1.75)$ \\
\hline Venting & $4.76(1.34)$ & $4.76(1.67)$ & $4.76(1.47)$ \\
\hline Positive reframing $\star \star$ & $5.24(1.60)$ & $5.65(1.77)$ & $5.39(1.67)$ \\
\hline Planning & $5.35(1.62)$ & $5.43(1.87)$ & $5.38(1.71)$ \\
\hline Humor** & $4.76(1.78)$ & $4.93(1.89)$ & $4.82(1.82)$ \\
\hline Acceptance ${ }^{\star \star}$ & $4.90(1.63)$ & $5.15(1.78)$ & $4.99(1.69)$ \\
\hline Religion** & $5.85(1.76)$ & $6.09(2.00)$ & $5.94(1.85)$ \\
\hline Self-blame** & $4.57(1.73)$ & 4.78 (1.64) & 4.65 (1.70) \\
\hline
\end{tabular}

\section{Key: -}

* Male mean scores are significantly higher than male mean scores.

** Female mean scores are significantly higher than female mean scores.

\section{Coping Strategies Adopted Under Depressed Situation}

A binary logistic regression analysis was done to assess common coping strategies that were utilized by respondents under depressed situation. From total 14 factors of the $B C$ instrument, 7 factors were not statistically associated with respondents' depression level $(p<0.05)($ Table 8). However, the coping mechanisms of denial, substance use, use of emotional support, the use of instrumental support, behavioral disengagement, venting, self-blame and humor were significant variations among DASdepression distribution. 
Table 8

Bivariate logistic regression of coping strategies adopted by respondents under depressed situation, $\mathrm{N}=260, \mathrm{AU}$, January 2019.

\begin{tabular}{|lllll|}
\hline Category & Overall & \multicolumn{2}{l}{ Depression } & \multirow{2}{*}{ P-value } \\
\cline { 3 - 4 } & Mean (SD) & Yes & No & \\
& & Mean (SD) & Mean (SD) & \\
\hline Self-distraction & $5.08(1.52)$ & $5.24(1.46)$ & $4.90(1.57)$ & 0.078 \\
\hline Active coping & $5.52(1.75)$ & $5.68(1.54)$ & $5.36(1.94)$ & 0.139 \\
\hline Denial & $4.10(1.70)$ & $4.54(1.75)$ & $3.62(1.52)$ & 0.001 \\
\hline Substance use & $3.47(1.81)$ & $2.99(1.55)$ & $3.91(1.92)$ & 0.001 \\
\hline Use of emotional support & $4.69(1.62)$ & $4.97(1.56)$ & $4.39(1.65)$ & 0.004 \\
\hline Use of instrumental support & $5.07(1.73)$ & $5.32(1.66)$ & $4.79(1.76)$ & 0.013 \\
\hline Behavioral disengagement & $4.34(1.75)$ & $4.88(1.68)$ & $3.75(1.65)$ & 0.001 \\
\hline Venting & $4.76(1.47)$ & $5.15(1.34)$ & $4.33(1.50)$ & 0.001 \\
\hline Positive reframing & $5.39(1.67)$ & $5.52(1.60)$ & $5.24(1.75)$ & 1.178 \\
\hline Self-blame & $4.65(1.70)$ & $5.20(1.62)$ & $4.05(1.57)$ & 0.001 \\
\hline Planning & $5.38(1.71)$ & $5.48(1.66)$ & $5.27(1.77)$ & 0.321 \\
\hline Humor & $4.82(1.82)$ & $5.13(1.68)$ & $4.49(1.91)$ & 0.005 \\
\hline Acceptance & $4.99(1.69)$ & $5.09(1.64)$ & $4.88(1.74)$ & 0.319 \\
\hline Religion & $5.94(1.85)$ & $5.88(1.84)$ & $6.00(1.87)$ & 0.610 \\
\hline
\end{tabular}

\section{Coping Strategies Adopted Under Anxious Situation}

In the present study, a binary logistic regression analysis was done to assess common coping strategies that were utilized by respondents under anxious situation. Out of 14 factors of the BC instrument, 8 factors were not statistically associated with the scores of respondents' anxiety level $(p<0.05)($ Table 9$)$. Nevertheless, the coping mechanisms of denial, substance use, behavioral disengagement, venting, positive reframing, and self-blame were significant variations among DAS-anxious distribution. 
Table 9

Bivariate logistic regression of coping strategies adopted by respondents under anxious situation, $\mathrm{N}=260, \mathrm{AU}$, January 2019.

\begin{tabular}{|lllll|}
\hline Category & Overall & Anxiety & & \multirow{2}{*}{ P-value } \\
\cline { 3 - 4 } & Mean (SD) & Yes & No & \\
& & Mean (SD) & Mean (SD) & \\
\hline Self-distraction & $5.08(1.52)$ & $5.23(1.51)$ & $4.84(1.50)$ & 0.050 \\
\hline Active coping & $5.52(1.75)$ & $5.64(1.57)$ & $5.34(1.99)$ & 0.183 \\
\hline Denial & $4.10(1.70)$ & $4.49(1.71)$ & $3.51(1.51)$ & 0.001 \\
\hline Substance use & $3.47(1.81)$ & $3.76(1.94)$ & $3.03(1.48)$ & 0.001 \\
\hline Use of emotional support & $4.82(1.60)$ & $4.49(1.67)$ & $4.70(1.63)$ & 0.108 \\
\hline Use of instrumental support & $5.07(1.73)$ & $5.24(1.67)$ & $4.80(1.79)$ & 0.050 \\
\hline Behavioral disengagement & $4.34(1.75)$ & $4.71(1.68)$ & $3.78(1.72)$ & 0.001 \\
\hline Venting & $4.76(1.47)$ & $5.01(1.39)$ & $4.36(1.51)$ & 0.001 \\
\hline Positive reframing & $5.39(1.67)$ & $5.56(1.65)$ & $5.13(1.69)$ & 0.043 \\
\hline Self-blame & $4.65(1.70)$ & $4.93(1.67)$ & $4.22(1.66)$ & 0.001 \\
\hline Planning & $5.38(1.71)$ & $5.54(1.67)$ & $5.12(1.76)$ & 0.050 \\
\hline Humor & $4.82(1.82)$ & $5.00(1.79)$ & $4.55(1.84)$ & 0.051 \\
\hline Acceptance & $4.99(1.69)$ & $5.02(1.67)$ & $4.94(1.72)$ & 0.717 \\
\hline Religion & $5.94(1.85)$ & $6.03(1.80)$ & $5.80(1.94)$ & 0.348 \\
\hline
\end{tabular}

\section{Coping Strategies Adopted Under Stressed Situation}

A binary logistic regression analysis was also done to assess common coping strategies that were utilized by respondents under stressed situation. Other than humor, acceptance and religion, all other factors of the BC instrument were statistically associated with respondents stress level $(p<0.05)$ (Table 10). 
Table 10

Bivariate logistic regression of coping strategies adopted by respondents under stressed situation, $\mathrm{N}=260$, AU, January 2019.

\begin{tabular}{|lllll|}
\hline Category & Overall & Stress & & \multirow{2}{*}{ P-value } \\
\cline { 3 - 4 } & Mean (SD) & Yes & No & \\
& & Mean (SD) & Mean (SD) & \\
\hline Self-distraction & $5.08(1.52)$ & $5.31(1.30)$ & $4.92(1.64)$ & 0.038 \\
\hline Active coping & $5.52(1.75)$ & $5.91(1.51)$ & $5.26(1.85)$ & 0.003 \\
\hline Denial & $4.10(1.70)$ & $4.55(1.81)$ & $3.80(1.56)$ & 0.001 \\
\hline Substance use & $3.47(1.81)$ & $3.77(2.06)$ & $3.27(1.59)$ & 0.028 \\
\hline Use of emotional support & $4.69(1.63)$ & $5.10(1.60)$ & $4.42(1.59)$ & 0.001 \\
\hline Use of instrumental support & $5.01(1.73)$ & $5.48(1.66)$ & $4.79(1.72)$ & 0.002 \\
\hline Behavioral disengagement & $4.34(1.75)$ & $4.86(1.80)$ & $3.99(1.64)$ & 0.001 \\
\hline Venting & $4.76(1.47)$ & $5.20(1.38)$ & $4.46(1.46)$ & 0.001 \\
\hline Positive reframing & $5.39(1.67)$ & $5.72(1.56)$ & $5.16(1.72)$ & 0.008 \\
\hline Self-blame & $4.65(1.70)$ & $5.34(1.46)$ & $4.18(1.69)$ & 0.001 \\
\hline Planning & $5.38(1.71)$ & $5.84(1.58)$ & $5.07(1.74)$ & 0.001 \\
\hline Humor & $4.82(1.82)$ & $5.06(1.75)$ & $4.67(1.85)$ & 0.088 \\
\hline Acceptance & $4.99(1.69)$ & $5.20(1.61)$ & $4.85(1.73)$ & 0.096 \\
\hline Religion & $5.94(1.85)$ & $6.16(1.77)$ & $5.79(1.90)$ & 0.110 \\
\hline
\end{tabular}

\section{Discussion}

The main goal and objective of medical curriculum is to provide competent and safe doctors to the community. However, mental illness among medical students has often been swept under the carpet and under-recognized, though the rates of these mental illnesses among this vulnerable population are by no means trivial. Therefore, the main aim of the present study was to determine the prevalence and severity of depression, anxiety and stress as well as coping strategies used by medical undergraduate students, enrolled in Arsi University.

The overall prevalence of depression, anxiety and stress symptoms were $52.3 \%, 60.8 \%$ and $40.4 \%$ respectively. The reason might be medical students are overloaded with a tremendous amount of information with limited time for internalization, new study environment with obligations to succeed especially during preclinical encounters. This greater degree of workload creates feeling of distress and disappointments that predisposes students to have difficulties in solving problems, impaired judgments; 
absenteeism from class lesson and break their mental stability. Actually, our present finding is almost similar to the prevalence reported by Basudan et $\mathrm{al}^{22}$, and Iqbal et $\mathrm{al}^{23}$. Conversely, it is higher than study report of Shamsuddin et $\mathrm{al}^{24}$, and Moutinho et $\mathrm{al}^{25}$. This difference could be due to difference in cultural perception of stressful factors, economic burden, very high tuition fees, lack of family support, and higher or lower 'readiness' to report different complaint. In our present setup, academic counselling is not a common practice which may be also a contributor.

The proportion of respondents who had extremely severe symptoms of depression, anxiety and stress were $6.2 \%, 16.2 \%$ and $2.3 \%$ respectively. This prevalence was similar with finding of Patil et $\mathrm{al}^{26}$. However, it is inconsistent with study result of Gan et $\mathrm{al}^{27}$ and Al-Ani Radeef and Ghazi ${ }^{28}$. Discrepancies stemming from methodology and type of questionnaire used, could account for this high prevalence obtained by the aforementioned authors. The other possible reasons for the variability could be due to certain differences in the curricula, teaching facilities, qualification and experience of the instructors, and levels of care given to the students.

In this cross-sectional study we correlated sociodemographic risk factors of the student (as independent variables) with the prevalence of depression, anxiety and stress level amongst the undergraduate medical students of Arsi University. In our study, students living in non-dormitory have considerably higher degree of depression, anxiety and stress, which infers living in dormitory, came out to be protective. This finding is in line with study reported by Rab et $\mathrm{al}^{29}$ and Shendarkar ${ }^{30}$. However, it is inconsistent with study report Kunwar et $\mathrm{al}^{31}$ and Liaqat et $\mathrm{al}^{32}$. This unequivocal distribution tells us that probably depression, anxiety and stress are associated with a multitude of factors such as poor dormitory conditions, more economic stress, distance from the family, less structured environment, and problems dealing with roommates, which might be different or parallel in the two study groups under consideration.

Medical students who had lower monthly income were more likely depressed and stressed. Despite the fact that food and dormitory services are provided to the students by the university, students need money for excursions, to print hand outs, to buy dressings, and other basic necessities ${ }^{11}$. This indicates that financial constraints could be an additional source of depression, anxiety and stress besides academic stressors. However, other studies have noted no difference ${ }^{33}$. Again, sample size differences may possibly account for these different results. The same stressors may be perceived differently by different medical students, depending on their cultural background, personal traits, experience and coping skills ${ }^{34}$. The educational system also plays an enabling role subsequently leading to increased stress levels experienced by students.

There was a significant association between students' academic year and level of anxiety and stress. Many studies have shown higher stress levels and a higher prevalence of stress-related illnesses among 2nd year medical students as compared with other academic years ${ }^{11,35}$. On the other hand, senior students developed skills of how to manage stress and stress-related illness than students in the early years ${ }^{36}$. The possible reason could be the amount and complexity of the material to be learned in the 
second year with progressive assessments of anatomy, physiology, and biochemistry that they have to pass to join the next higher level. Additional supportive evidence is high level of stress and stress-related illness can be attributed by course workload, lack of leisure time, shortage of learning materials, and frequent examinations.

It has been proven that coping mechanisms are essential for individuals perceiving stress. Coping strategies refer to specific efforts, both behavioral and psychological, that people employ to master, reduce, tolerate, or minimize stress due to undesired events ${ }^{37}$. Effective and appropriate coping strategies may minimize the impact of encountered stressful situations on one's well-being. The strategies that the students identified for coping with stress covered almost all the categories reported previously. 'Active coping' means taking action or exerting efforts to remove or circumvent the stressor, while 'Acceptance' means accepting the stressful event, 'Planning' consists of thinking about how to confront the stressor, 'Positive' reframing means making best of the situation by growing from it and 'Denial' is an attempt to reject the reality of stressful event and 'Behavioral disengagement' means giving up or withdrawing efforts to attain goal ${ }^{13}$.

In the present study, the widely employed original COPE questionnaire containing 14 domains of coping strategies was utilized in order to assess the most common strategies adopted by respondents. The coping strategies most commonly reported by respondents comprise religious coping, active coping, positive reframing and planning strategies. The main coping strategy adopted by respondents was religion, which was similar with the study by Al-Sowygh ${ }^{38}$, and Ahmad et al ${ }^{39}$. This was also reported by Krauss et $\mathrm{al}^{40}$, stated that religious people posed higher level self-control thus they are more able to persist in difficult tasks and life situation. However, our result finding was dissimilar with study finding reported in Malaysia ${ }^{41}$, and Jordan ${ }^{42}$. The possible reasons for the variability could be due to certain differences in the curricula, teaching facilities and the levels of care given to the students.

A significant correlation between DAS scores and coping mechanisms was found. In particular, the coping mechanisms of denial, substance use, behavioral disengagement, venting, self-blame were significantly associated to DAS, which have been reported in studies as very adaptive and hasten the recovery from distress ${ }^{38}$. However, the present study was dissimilar with studies conducted on the United Kingdom and Jordanian medical students, who are using alcohol, tobacco, and drugs as common coping strategies ${ }^{7,8}$. The possible reasons for the variability could be due to geographical and racial differences.

\section{Conclusion}

Healthy medical students are likely to become healthy doctors. However, the overall prevalence of depression, anxiety and stress is alarmingly high among undergraduate medical students of Arsi University. Furthermore, monthly income and residency were identified as risk factors of depression. Similarly, residency and educational level were risk factors for anxiety. Lastly, monthly income, educational level and residency were predictors for stress. There was no significant difference in depression, anxiety and stress between age groups, sex, marital status, ethnicity and religion. It has been 
suggested that medical educators should include student-centered academic policies, non-quantitative evaluation of training, feed-back and advisory systems for students and overall improvement of the learning environment. Also, special attention needs to be given to stress reduction. It is noteworthy that, despite positive coping strategies used by the students, the stress prevalence is still higher from the normal population. Perhaps, it will be interesting to explore this matter further in future studies. Further studies are also needed to evaluate the effects of support mechanisms and the strategies provided by the University.

\section{Abbreviations}

ARS - Academic related stressors

AOR - Adjusted odds ratio

COR - Crudes odds ratio

$\mathrm{Cl}$ - Confidence interval

DRS - Desire and drive related stressors

Epi Data - Epidemiological Data

ETB - Ethiopia Birr

GARS - Group activities related stressors

IRS - Interpersonal and intrapersonal related stressors

MSSQ - Medical Student Stressor Questionnaires

SD - Standard deviation

SRS - Social related stressors

SPSS - Statistical Package for Social Science

TLRS - Teaching and learning related stressors

\section{Declarations}

\section{AVAILABILITY OF DATA AND MATERIALS}

The datasets used and/or analyzed during the current study are available from the corresponding author on reasonable request. 


\section{ETHICAL ISSUE}

Ethical approval was obtained from Arsi University Ethical Review Committee. Written informed consent was taken from the students during data collection. The confidentiality was kept anonymous.

\section{FUNDING}

Nil support in financial or other manner

\section{COMPETING INTERESTS}

The authors declare that there is no conflict of interests regarding the publication of this paper.

\section{AUTHORS' CONTRIBUTIONS}

LM, GB and DW had participated in the design of the study data analyses and manuscript preparation. All authors have equal contribution; and all authors have read and approved the final manuscript.

\section{ACKNOWLEDGEMENT}

Authors are grateful to College of Health Sciences Research and Community Office of Arsi University, all medical students of Arsi University, data collectors as well as supervisors for their support and contribution.

\section{RIGHTS AND PERMISSIONS}

Open Access: This article is licensed under a Creative Commons Attribution 4.0 International License, which permits use, sharing, adaptation, distribution and reproduction in any medium or format, as long as you give appropriate credit to the original author(s) and the source, provide a link to the Creative Commons licence, and indicate if changes were made. The images or other third party material in this article are included in the article's Creative Commons licence, unless indicated otherwise in a credit line to the material. If material is not included in the article's Creative Commons licence and your intended use is not permitted by statutory regulation or exceeds the permitted use, you will need to obtain permission directly from the copyright holder. To view a copy of this licence, visit http://creativecommons.org/licenses/by/4.0/. The Creative Commons Public Domain Dedication waiver (http://creativecommons.org/publicdomain/zero/1.0/) applies to the data made available in this article, unless otherwise stated in a credit line to the data.

\section{References}

1. Goldberger, L. \& Breznitz, S. Handbook of stress: Theoretical and Clinical aspects. (Free Press, 1987).

2. Radeef, A., Faisal, G., Ali, S. \& Ismail, M. Source of stressors and emotional disturbances among undergraduate science students in Malaysia. Int J Med Res Health Sci3, 401-410 (2014). 
3. Marcus, M., Yasamy, M., van Ommeren, M., Chisholm, D. \& Saxena, S. Depression: A global public health concern. in vol. 1 6-8 (WHO, Department of Mental Health and Substance Abuse., 2012).

4. Dyrbye, I., Thomas, M. \& Shanafelt, T. Systematic review of depression, anxiety, and other indicators of psychological distress among U.S and Canadian medical students. Acad Med81, 354-373 (2006).

5. Nechita, F., Strba, C., Vere, C., Nechita, D. \& Rogoveanu, I. Stress in Romanian first year nursing students. Curr Health Sci J40, 210-213 (2014).

6. Sarkar, S., Gupta, R. \& Menon, V. A systematic review of depression, anxiety, and stress among medical students in India. J Mental Health Hum BehaV22, 88-96 (2017).

7. Guthrie, E. et al. Embarking upon a medical career: Psychological morbidity in first year medical students. Med Educ29, 337-341 (1995).

8. Firth, J. Levels and sources of stress in medical students. Br Med J292, 1177-1180 (1986).

9. Sherina, M., Rampal, L. \& Kaneson, N. Psychological stress among undergraduate medical students. Med J Malaysia59, 207-211 (2004).

10. Oku, A., Owoaje, E., Oku, O. \& Ikpeme, B. Prevalence of stress, stressors and coping strategies among medical students in a Nigerian medical school. Afr J Med Health Sci14, 29-34 (2015).

11. Melaku, L., Mossie, A. \& Negash, A. Stress among Medical Students and Its Association with Substance Use and Academic Performance. J Biomed Educ2015, 1-9 (2015).

12. Agrawal, R. \& Chahar, S. Examining role stress among technical students in India. Social Psychology of Education10, 77-91 (2007).

13. Sreeramareddy, C. et al. Psychological morbidity, sources of stress and coping strategies among undergraduate medical students of Nepal. BMC Med Educ7, 26 (2007).

14. Deb, S., Strodl, E. \& Sun, J. Academic stress, parental pressure, anxiety and mental health among Indian high school students. International Journal of Psychology and Behavioral Sciences5, 26-34 (2015).

15. Shakthivel, N. et al. Level of perceived stress and coping strategies prevailing among 1 st year medical undergraduate students: A cross-sectional study from south India. Int J Med. Public Health7, 111-115 (2017).

16. Dahlin, M., Joneborg, N. \& Runeson, B. Stress and depression among medical students: a crosssectional study. Med Educ39, 594-604 (2005).

17. Memon, A. et al. Perceived stress among physical therapy students of Isra university. Int J Physiother3, 35-38 (2016).

18. Crawford, J. \& Henry, J. The Depression Anxiety stress scales (DASS): Normative data and latent structure in a large nonclinical sample. Br J Clin Psychol42, 111-131 (2003).

19. Vignola, R. \& Tucci, A. Adaptation and validation of the depression, anxiety and stress scale (DASS) to Brazilian Portuguese. J Affect Disord155, 104-109 (2014).

20. Silva, H. et al. Short version of the Depression Anxiety Stress Scale-21: is it valid for Brazilian adolescents? Einstein14, 486-493 (2016). 
21. Carver, C. You want to measure coping but your protocol's too long: Consider the brief COPE. Int $J$ Behav Med4, 92-100 (1997).

22. Basudan, S., Binanzan, N. \& Alhassan, A. Depression, anxiety and stress in dental students. International Journal of Medical Education8, 179-186 (2017).

23. Iqbal, S., Gupta, S. \& Venkatarao, E. Stress, anxiety and depression among medical undergraduate students and their socio-demographic correlates. Indian J Med Res141, 354-357 (2015).

24. Shamsuddin, K. et al. Correlates of depression, anxiety, and stress among Malaysian university students. Asian J Psychiatr6, 318-323 (2013).

25. Moutinho, I. et al. Depression, stress and anxiety in medical students: A cross-sectional comparison between students from different semesters. Rev Assoc Med Bras63, 21-28 (2017).

26. Patil, S., Sadhanala, S. \& BansodeGokhe, S. Study of Depression, Anxiety and Stress among Undergraduate Medical Students of A Teaching Medical Institution. Natl J Community Med9, 566569 (2018).

27. Gan, W., Mohd Nasir, M., Shariff, Z. \& Saad, H. Disordered eating behaviours, depression, anxiety and stress among Malaysian university students. Coll Stud J45, 296 (2011).

28. Al-Ani Radeef, A. \& Ghazi, F. Depression, Anxiety and Stress among Undergraduate Science Students in Malaysia. (2015).

29. Rab, F., Mamdou, R. \& Nasir, S. Rates of depression and anxiety among female medical students in Pakistan. East Mediterr Health J14, 126-133 (2008).

30. Ajay, S. \& Vijay, P. A study of stressors in medical college students (Hostilities) in Northern Maharashtra. J Indian Acad Forensic Med35, 227-229 (2013).

31. Kunwar, D., Risal, A. \& Koirala, S. Study of depression, anxiety and stress among the medical students in two medical colleges of Nepal. Kathmandu Univ Med J53, 22-26 (2016).

32. Liaqat, H. et al. Deranged Mental Homeostasis in Medical Students: Evaluation of Depression Anxiety and Stress among Home and Hostel Students. Acta Psychopatho/3, 1 (2017).

33. Koochaki, G. et al. Prevalence of stress among Iranian medical students: a questionnaire survey. East Mediterr Health J17, 593-598 (2011).

34. Yusoff, M. A multi-center study on validity of medical student stressor questionnaire (MMS). International Medical Journal18, 14-18 (2011).

35. Khan, M., Mahmood, S., Badshah, A., Ali, S. \& Jamal, Y. Prevalence of depression, anxiety and their associated factors among medical students in Karachi, Pakistan. J Pak Med Assoc56, 583-586 (2006).

36. Alvi, T., Assad, F., Ramzan, M. \& Khan, F. Depression, Anxiety and Their Associated Factors Among Medical Students. Journal of the College of Physicians and Surgeons Pakistan20, 122-126 (2010).

37. Myers, D. Stress and Health. (Worth Publishers, 2005).

38. Al-Sowygh, Z. Academic distress, perceived stress and coping strategies among dental students in Saudi Arabia. The Saudi Dental Journa/25, 97-105 (2013). 
39. Ahmad, M., Ishak, A. \& Mohd, M. Stress and its relief among undergraduate dental students in Malaysia. Southeast Asian J Trop Med Public Health42, 996-1004 (2011).

40. Krauss, S. Development of the muslim religiosity- personality inventory for measuring the religiousity of Malaysia muslim youth. (Universiti Putra Malaysia, 2005).

41. Al-Dubai, S., Al-Naggar, R., Mustafa, A. \& Krishna, G. Stress and coping strategies of students in a medical faculty in Malaysia. Malaysian Journal of Medical Science18, 57-64 (2011).

42. Bataineh, Z., Hijazi, T. \& Hijleh, M. Attitudes and reactions of Jordanian medical students to the dissecting room. Surgical and Radiologic Anatomy28, 416-421 (2006). 